\title{
Terapia de Interacción Guiada: Una Nueva Modalidad de Intervención con Familias Multiproblemáticas y en Riesgo Social ${ }^{1}$
}

\author{
Interaction Guidance Therapy: a new form of intervention \\ with multiproblem families in social risk
}

\author{
Nicolás Suárez Delucchi Magdalena Muñoz Quinteros Esteban Gómez Muzzio \\ Sociedad Protectora de la Infancia, Chile \\ María Pía Santelices Álvarez \\ Pontificia Universidad Católica de Chile, Chile
}

(Rec: 14 de diciembre 2007 Acep: 1 junio 2009)

\begin{abstract}
Resumen
El artículo presenta una nueva forma de intervención terapéutica con familias multiproblemáticas, denominada Terapia de Interacción Guiada (IG) e ilustra su aplicación mediante un estudio de caso, llevado a cabo en un programa social de la Sociedad Protectora de la Infancia de Santiago de Chile. La IG es una terapia breve que, desde el enfoque sistémico y la teoría del apego, se focaliza en mejorar las interacciones cuidador(a)-niño(a). La IG intercala sesiones de juego familiar grabadas en video, con sesiones de observación de secuencias de interacción positivas y negativas escogidas por el terapeuta. Se discute su potencial beneficio con estas familias y los desafíos que implica adaptar e implementar este modelo al contexto chileno.

Palabras claves: Interacción guiada, familias multiproblemáticas, intervención temprana, salud mental infantil, video-retroalimentación.
\end{abstract}

\begin{abstract}
The paper present a new form of intervention with multiproblem families called Interaction Guidance Therapy (IG) and illustrate it's application trough a description of one therapy process developed on a social program of Sociedad Protectora de la Infancia. IG is a brief therapy that, from attachment and system theories, focuses on enhanced caregiver-child interaction. IG combines family play sessions recorded on video, with sessions of discussion about whose objective is to show and to discuss positive and negative interaction sequences chosen by the therapist. Potential benefits with this families and challenges that implied to adapt and to implement a model of this kind in social organizations and health services in Chile are discussed.

Key words: Interaction guidance, multiproblem families, early intervention, infant mental health, video feedback.
\end{abstract}

\footnotetext{
1 Correspondencia: nicolasuarez@gmail.com (nsuarez@uc.cl)o a egomez@ protectora.cl. Agradecimientos al Proyecto FONDECYT Nº1070839.
} 


\section{Introducción}

Los programas sociales y los servicios de salud se ven enfrentados muchas veces al complejo desafío de ayudar a familias altamente vulnerables y en riesgo social. Un gran porcentaje de estas familias corresponde a las características que se han asociado en la literatura al concepto de "familias multiproblemáticas", es decir, familias con poli-sintomatología y crisis recurrentes, desorganización estructural y comunicativa, tendencia al abandono de sus funciones parentales y aislamiento social (Gómez, Muñoz \& Haz, 2007).

Otra forma de conceptualizar estos sistemas familiares es como familias sobre-exigidas por múltiples factores de riesgo y estrés (Sharlin \& Shamai, 1995), situándolas en una mayor vulnerabilidad para desarrollar problemas graves, que en numerosas ocasiones resultan en vulneraciones de derecho para la infancia. La intervención con estos grupos familiares es de alta complejidad y se constituye en un desafío constante para los equipos profesionales, requiriendo intervenciones integrales y multimodales, que vinculen al sistema familiar con el sistema legal, educacional, los servicios de protección y el sistema de salud, entre otros (Larrieu \& Zeanah, 2004).

En este contexto, la terapia de Interacción Guiada (IG) se ha convertido en una herramienta clínica a implementar en programas psicosociales que abordan la relación padrehijo o cuidador-niño en contextos de maltrato infantil y familias multiproblemáticas (Larrieu \& Zeanah, 2004). La IG es una terapia breve centrada en las interacciones padreshijo(a), que busca reforzar los comportamientos interactivos funcionales y modificar los patrones disfuncionales, particularmente aquellos relacionados a los síntomas que presenta el niño(a), los cuales constituyen el foco terapéutico (McDonough, 1995). El trabajo terapéutico se basa en la filmación de secuencias de interacción y el posterior análisis de las filmaciones con los padres, llevándolos a identificar sus propios recursos, reconociendo en los padres el rol de expertos de sus propios hijos (Rusconi-Serpa, 1992).

El presente artículo, entonces, presenta el modelo de la IG e ilustra su implementación con un caso de familia multiproblemática en un programa de la Sociedad Protectora de la Infancia de Chile.

\section{Antecedentes}

La IG corresponde a una modalidad de terapia breve enmarcada en la corriente de intervenciones e investigaciones que en los últimos años han dado cuerpo a una práctica profesional especialmente diseñada para el abordaje de los desafíos específicos de la salud mental infantil en diversos contextos. La IG tiene diversos antecedentes teóricos, y sus influencias pueden rastrearse a modelos tanto psicoanalíticos como sistémicos de intervención.
A nivel teórico, la IG resulta altamente compatible con los postulados del Modelo Interaccional de Bromwich (1990). Este enfoque propone dos principios fundamentales a tomar en cuenta: (a) que tanto el niño como el padre/ madre o cuidador contribuyen a lo que ocurre entre ellos y (b) que múltiples factores, internos y ambientales, afectan la interacción entre ellos (Bromwich, 1990).

A nivel metodológico, la IG muestra alta congruencia con las intervenciones orientadas a la prevención, tratamiento y reparación de temáticas ligadas al apego en la temprana infancia (Bakermans-Kranenburg, van IJzendoorn \& Juffer, 2005; Juffer, Bakermans-Kranenburg y van IJzendoorn, 2005). Actualmente, ese campo se ha expandido notoriamente, para incluir nuevos desafíos, temáticas relacionadas y diferentes etapas del ciclo vital. La IG resulta altamente congruente con lo que nos informa la literatura especializada en este campo, por ejemplo, al utilizar técnicas de retroalimentación de las fortalezas y aspectos a mejorar en la interacción padre-hijo, al permitir la vinculación entre las representaciones de los cuidadores y la relación vincular actual con el niño(a), y al facilitar la estimulación de la sensibilidad materna y la capacidad de mentalización en los cuidadores (Juffer et al., 2005; O'Connor y Zeanah, 2003; Bakermans-Kranenburg et al., 2005; BakermansKranenburg, Juffer \& van IJzendoorn, 1998). Sin embargo, aun cuando la IG es compatible con las directrices señaladas para el abordaje de problemas de apego en infantes y niños pequeños, no se limita sólo a este campo de acción, permitiendo la intervención en un ámbito mucho más amplio de temáticas relacionadas a la parentalidad y el desarrollo saludable de la infancia, no solamente de los bebés.

\section{La Interacción Guiada}

La terapia IG fue creada en Estados Unidos por la trabajadora social especializada en investigación e intervención clínica con familias vulnerables, Susan McDonough, con la finalidad de ofrecer una herramienta técnica especialmente diseñada para trabajar con casos resistentes a las formas tradicionales de abordaje clínico, en programas sociales y servicios de salud. Es así que la IG fue pensada como una forma alternativa de intervención con familias sobreexigidas por múltiples problemas y factores de riesgo, que mostraban resistencia a otros enfoques (McDonough, 1993; 2004). Es conocido lo difícil que resulta para los equipos profesionales el abordaje exitoso de los desafíos que implica trabajar con estas familias, tanto en términos de su conceptualización, involucramiento y permanencia en el proceso de intervención, generación de cambios en sus pautas de interacción y mantención de los cambios en el tiempo (Gómez et al., 2007; McNeil \& Herschell, 1998; Cunningham \& Henggeler, 1999). El método propuesto por McDonough (1995), es un aporte sustantivo al campo de la acción profesional en comunidades con altos índices de riesgo social (Larrieu \& Zeanah, 2004). 
Cabe destacar que a diferencia de otras formas ampliamente conocidas de intervención clínica infantil, como la psicoterapia padre-infante (Lieberman, 2004), la Interacción Guiada no se dirige específicamente a la búsqueda del insight como mecanismo generativo de cambios en la relación padre-hijo, sino más bien al refuerzo de los patrones y competencias saludables de la interacción y a la modificación de las pautas interaccionales disfuncionales de la relación (McDonough, 1993; 2004). En este sentido, la IG tiene el objetivo de ayudar a los padres a reconocer y desplegar sus capacidades y a sentirse más competentes, a disfrutar más de la relación con su hijo/a, y a desarrollar una mayor comprensión de sus comportamientos, recursos y necesidades ligadas a su desarrollo evolutivo y características particulares (Forcada-Guex, 2007).

\section{Indicaciones}

Según Forcada-Guex (2007), la IG se indica tras la detección de interacciones familiares disfuncionales o poco funcionales, para dificultades o síntomas diversos infantiles, y para madres adolescentes, con poca experiencia y/o madres depresivas. También se indica frente a padres que muestren un grado elevado de ansiedad respecto a su nivel de competencia parental.

Por otra parte, la IG se indica para padres y madres con bajo nivel de "insight" (y resistentes por tanto a formas tradicionales de psicoterapia) o con limitaciones intelectuales
(Larrieu \& Zeanah, 2004). Sin embargo, no sólo se indica para situaciones difíciles o complejas, sino que también frente a adultos interesados en poder reflexionar sobre su parentalidad, en el extremo más preventivo de las posibilidades de intervención (Forcada-Guex, 2007).

\section{Metodología}

La particularidad de este tratamiento radica en grabar en video, en cada sesión, una secuencia de juego de algunos minutos. El terapeuta le pide a los padres que jueguen como si estuvieran en su casa, utilizando un set de juguetes adecuados a la edad del niño(a). Durante el juego libre el terapeuta se queda en la sala retirado de la interacción con una actitud de observación neutra (Rusconi-Serpa, 1992). La IG intercala estas sesiones de juego familiar grabadas en video, con sesiones de exposición y discusión (retroalimentación) de secuencias de interacción positivas y negativas, escogidas por el terapeuta, ayudando a los padres o cuidadores a mejorar su comprensión del hijo(a), identificar y reforzar sus propias competencias, a disfrutar de la relación con su hijo(a) y a mejorar el vínculo afectivo entre ambos (McDonough, 1993; Forcada-Guex, 2007). La selección de las secuencias de video se basa en el foco de intervención desarrollado por el terapeuta, en base a las hipótesis que ligan las dificultades interactivas observadas con los motivos que originan la terapia (Forcada-Guex, 2007).

Tabla 1: Principios, Implementación, Indicaciones y Resultados de la IG

\section{CARACTERÍSTICAS DE LA INTERACCIÓN GUIADA}

\begin{tabular}{|c|c|c|c|}
\hline $\begin{array}{c}\text { Principios que orientan la } \\
\text { terapia }\end{array}$ & $\begin{array}{l}\text { Elementos para la } \\
\text { implementación }\end{array}$ & Indicaciones & Resultados demostrados \\
\hline $\begin{array}{l}\text { Intervención breve } \\
\text { Énfasis en las fortalezas y } \\
\text { recursos } \\
\text { Énfasis en las interacciones aquí } \\
\text { y ahora } \\
\text { Uso de un foco de intervención } \\
\text { Video-Retroalimentación de lo } \\
\text { que sí funciona }\end{array}$ & $\begin{array}{l}\text { Cámara video-grabadora } \\
\text { con trípode } \\
\text { Caja de juguetes } \\
\text { Mesa o alfombra } \\
\text { Sala adecuada } \\
\text { Reproductor de video o PC }\end{array}$ & $\begin{array}{l}\text { Interacciones disfuncionales } \\
\text { Diversos síntomas o proble- } \\
\text { mas en niños e infantes } \\
\text { Madres adolescentes o con } \\
\text { poca experiencia } \\
\text { Madres depresivas } \\
\text { Cuidadores ansiosos respecto } \\
\text { a su competencia } \\
\text { Cuidadores con limitaciones } \\
\text { cognitivas } \\
\text { Padres y madres con interés } \\
\text { por mejorar su competencia } \\
\text { parental }\end{array}$ & $\begin{array}{l}\text { En el sistema familiar } \\
\text { - Mejorías significativas en la interacción y } \\
\text { comunicación familiar } \\
\text { En el infante, niño o niña } \\
\text { - Efectiva en problemas de alimentación } \\
\text { en infantes } \\
\text { - Efectiva en desórdenes conductuales } \\
\text { - Otros problemas ligados a la relación con } \\
\text { su cuidador o cuidadores primarios }\end{array}$ \\
\hline
\end{tabular}


La IG encarna varios de los principios que se han propuesto en la literatura especializada como claves para avanzar en los desafíos profesionales propios del trabajo con familias altamente exigidas por múltiples factores de estrés (véase Gómez et al., 2007): (a) tiene un fuerte énfasis en las fortalezas y recursos de las personas, por sobre la consideración de carencias, limitaciones o patologías, permitiendo en muchos casos generar cambios puntuales en las interacciones familiares aun en presencia de múltiples problemas de otra índole; (b) se concentra en las interacciones sistémicas del aquí y ahora, por sobre referencias a los recuerdos de la historia pasada del cuidador (aunque el mundo interno de fantasías, sentimientos y representaciones puede ser explorado brevemente si emerge como un aspecto relevante para el tratamiento de un problema determinado); (c) utiliza el concepto de foco de intervención, permitiendo priorizar las necesidades de intervención y evaluar las posibilidades de cambio, acotando las energías profesionales a lo que puede ser cambiado y evitando disgregarse y confundirse con las múltiples problemáticas que usualmente presentan estas familias en los programas sociales; (d) se basa en la retroalimentación constante de los aspectos que sí funcionan en la interacción entre padres e hijos, sobretodo en una primera fase, generando una sólida alianza de trabajo que permita con posterioridad abordar los aspectos negativos de la interacción que requieren ser mejorados; (e) se plantea como una intervención breve, que en promedio puede durar entre 6-12 sesiones, por lo que los principios previamente mencionados resultan fundamentales si ha de lograrse el objetivo de modificar la interacción en un corto período de tiempo (McDonough, 2004).

\section{Implementación}

La Interacción Guiada puede realizarse tanto en el espacio físico de un centro de intervención clínica o psicosocial, como en el hogar de la familia mediante visitas domiciliarias, siempre y cuando el equipo profesional cuente con las herramientas indispensables para su implementación. Así, dentro de los elementos principales se encuentra el disponer de una cámara videograbadora, un trípode para encuadrar adecuadamente la imagen, una caja con diversos juegos y juguetes adecuados a la edad y cultura del niño o niña con quien se realizará la IG e idealmente una mesa o alfombra para el desarrollo del juego. Es indispensable contar a la vez con una sala o habitación adecuada que sea posible separar de las interferencias ambientales habituales durante el juego (llamados telefónicos, televisor, conversaciones, etc.). Además, se necesitará un reproductor de video o un computador con software que permita reproducir, detener, adelantar y/o retroceder las secuencias de video seleccionadas.

\section{Resultados demostrados}

La Interacción Guiada ha demostrado ser especialmente exitosa para obtener mejorías en la sensibilidad materna o la sensibilidad del cuidador (Robert-Tissot, et al., 1996), posiblemente debido a su énfasis en la observación de secuencias de interacción positivas y negativas, y su generación de preguntas orientadas a estimular la reflexión empática de los cuidadores respecto a los comportamientos del niño y los patrones de la relación, elemento que la investigación ha demostrado es de vital importancia para el desarrollo infantil, por tanto se liga a la capacidad de mentalización (Oppenheim, Goldsmith \& Koren-Karie, 2004; Fonagy et al., 1995a). Así, la IG tendría un elemento técnico central que consistiría en la generación de una dinámica clínica que estimula la sensibilidad y la función reflexiva parental, facilitando con posterioridad la generación de conductas sensibles en la relación, lo que se ha visto tiene un impacto significativo en diversas áreas del desarrollo infantil, especialmente en el desarrollo de un apego seguro (Kivijärvi et al., 2004; Vereijken, Riksen-Walraven \& Kondo-Ikemura, 1997).

En esta línea, la IG ha probado obtener notorios resultados en infantes con problemas de alimentación, permitiendo mejorar de forma significativa la sensibilidad de los cuidadores, disminuyendo la comunicación disruptiva entre madres y sus bebés (Benoit et al, 2001).

La IG ha mostrado asimismo ser útil para el abordaje de los problemas asociados a Síndrome de Estrés Posttraumático (EPT) en madres que han sufrido una historia de violencia y malos tratos (Schechter, 2004), permitiendo mejorar estilos de relación madre-hijo fuertemente "contaminados" por las consecuencias de dicho traumatismo. En esta línea, la IG demostró ser una herramienta muy efectiva para reducir el grado de distorsión negativa de las atribuciones maternas con respecto a su relación actual (Schechter, 2004).

Recientemente, el rango de aplicaciones de la IG ha comenzado a ampliarse a usuarios distintos de la díada madre-bebé. Por ejemplo en Escocia, en el contexto de la Inclusive Education, existe experiencia en el uso de la IG con niños en edad escolar. La IG es usada para trabajar en el contexto educativo con alumnos y profesores, mejorando la interacción y el manejo de los profesores con sus estudiantes (Forsyth, Kennedy \& Simpson, 1996; Forsyth \& Simpson, 2002). En línea con las nuevas aplicaciones de esta terapia, Simpson (2001) da cuenta de una intervención en que la IG fue usada para mejorar la comunicación entre hijos adolescentes que habían estado institucionalizados y sus padres, es decir, como parte de servicios posteriores a un proceso de reunificación familiar.

Si bien la IG ha demostrado obtener resultados por sí misma, diversos estudios apuntan a que su efectividad sería mayor cuando es usada en conjunto con otras modalidades 
de intervención. Así, cuando se ha implementado sobre la base de visitas domiciliarias orientadas a generar apoyo social, ha logrado mejorar la sensibilidad de los cuidadores, fortalecer el apego y abrir caminos de resiliencia en niños de comunidades altamente vulnerables (Svanberg, 2005). Además, la IG ha sido usada en conjunto con intervenciones de corte más psicodinámico, demostrando su efectividad, por ejemplo, para ligar aspectos significativos de la historia pasada del cuidador con las dificultades presentes observadas en las interacciones "videadas" entre madres e infantes (Beebe, 2003; Karlsson \& Skagerberg, 1999).

Por último, es relevante destacar que la IG ha sido usada exitosamente en un programa desarrollado por Larrieu y Zeanah (2004) en Estados Unidos, llamado Equipo Infancia (Infant Team), que incluye tanto a profesionales de un programa de Familias de Acogida como de una Universidad, recibiendo sus derivaciones de los Servicios de Protección Infantil, e iniciando su atención por mandato legal de una Corte Juvenil. Este programa se focaliza en desarrollar la reunificación de niños separados de su familia de origen por vulneración grave de derechos, con sus padres biológicos, después de una evaluación que indica la viabilidad y adecuación de dicha intervención. El programa ofrece y coordina intervenciones legales, sociales y clínicas; siendo una de las principales intervenciones clínicas implementadas, la terapia de Interacción Guiada. Larrieu y Zeanah (2004) señalan que la IG se usa especialmente en casos en los que los padres son jóvenes, cognitivamente limitados y/o emocionalmente cerrados debido a traumas crónicos en su historia de vida. El programa ha evaluado resultados en una cohorte de 4 años, encontrando cambios significativos en los niños atendidos, demostrando una reducción del $68 \%$ en la reincidencia de maltrato en el mismo niño, y de $75 \%$ para un niño nacido posteriormente de la misma madre (Zeanah et al., 2001, en Larrieu \& Zeanah, 2004).

El hecho de que la IG haya demostrado ser más efectiva en poblaciones vulnerables cuando es usada en conjunto con otras estrategias de intervención, probablemente se ligue a la constatación teórica y empírica de la necesidad de desarrollar intervenciones integrales para poder hacer frente a la complejidad multi-factorial del trabajo con familias multiproblemáticas y en riesgo social (Gómez et al., 2007; Larrieu \& Zeanah, 2004).

El caso particular que se presentará a continuación, corresponde a un proceso de reunificación familiar, de un niño egresado desde una Residencia de Protección de la SPI hacia su familia de origen. En este contexto, la técnica IG fue empleada como una de las intervenciones de apoyo psicoterapéutico al proceso de reunificación familiar, de forma similar al proceso desarrollado por Larrieu y Zeanah (2004) en su programa Equipo Infancia.

\section{Ilustración de la técnica a partir de un caso:}

El caso que aquí se presenta ilustra el uso de la IG en niños de edad escolar, ampliando el rango de uso predominante de la IG, registrado en la literatura, como herramienta de intervención temprana. Este caso clínico permite ilustrar el uso de la técnica dentro del contexto de intervención de la Sociedad Protectora de la Infancia (SPI) ${ }^{2}$, en un programa de Familias de Acogida (también conocidos como Colocación o Acogimiento Familiar) colaborador del Servicio Nacional de Menores de Chile. Estos programas se orientan a brindar protección, en un entorno familiar, a niños separados de su familia de origen por vulneración grave de derechos y a la espera de una solución definitiva (SENAME 2007).

Se expondrá primero una síntesis de los antecedentes del caso. Posteriormente, a modo de ilustración de la técnica, serán presentadas las 2 primeras sesiones del proceso de un total de 9, para finalmente señalar los logros con que se cerró el caso. Los nombres han sido modificados, al igual que algunos datos del caso, para resguardar los aspectos éticos en esta publicación.

\section{Antecedentes del caso}

El grupo familiar actual está compuesto por tres miembros: Sara, la madre, de treinta años, y sus hijos, Felipe, de siete años, y Alicia, de nueve. A principios del año en curso, y luego de 5 años de internación, Felipe egresa de la residencia institucional y se incorpora a vivir con su madre y hermana.

Esta familia se caracterizaba por ser multiproblemática y en riesgo social (Gómez et al., 2007), presentando una historia transgeneracional de daños vinculares y episodios traumáticos, violencia intrafamiliar, figuras parentales negligentes y abandono de sus funciones parentales, en un contexto de deprivación socioeconómica. Además, se observaba una desorganización estructural y comunicación disfuncional que dificultaba el proceso de reunificación familiar.

Como antecedentes relevantes de la historia familiar, cabe señalar la presencia en la madre de abuso sexual infantil intrafamiliar y abandono temprano de ambos padres. A los 19 años de edad, se registra un embarazo imprevisto que la madre asume sola en una primera etapa. Al año de vida de su hija Alicia, comienza una relación con Osvaldo, quien reconoce legalmente a Alicia. El segundo hijo de

\footnotetext{
2 La Sociedad Protectora de la Infancia es una organización sin fines de lucro fundada en 1894, y que durante 113 años de historia ha estado dedicada a mejorar las condiciones de la infancia en Chile, en particular de aquellos niños, niñas y jóvenes en mayor situación de vulnerabilidad y riesgo social. Tiene dos grandes áreas de servicios, educación y social; en su área social trabaja en la actualidad con más de 1.500 niños en situación de riesgo o desprotección social, en diversas líneas de servicios: residencias de protección, familias de acogida, programas de intervención breve, programa de reparación y programa de adopción (Protectora de la Infancia, 2007).
} 
Sara, Felipe, nace en el contexto de violencia intrafamiliar y violencia sexual íntima ejercida por Osvaldo; es así como la madre señala: "Felipe fue producto de una violación".

Luego de cuatro años de convivencia con su pareja, Sara se separa de Osvaldo aludiendo a los repetidos episodios de violencia intrafamiliar. En ese contexto, decide institucionalizar a su hijo Felipe a partir del año y medio y hasta los siete años de edad. Durante este periodo, Felipe tuvo posibilidades de ser declarado susceptible de adopción, ya que Sara no era considerada por el equipo profesional de la residencia como una madre competente para hacerse cargo de él. Su hija Alicia también estuvo institucionalizada entre los 6 y 7 años de edad, pero el resto del tiempo permaneció junto a Sara.

Durante la estadía de sus hijos en las instituciones, Sara presentó problemas con los profesionales en diversas oportunidades, llegando a realizar "denuncias por mala atención". Esto fue otro elemento de complejidad en el caso, ya que Sara tendía a generar una dinámica entre los profesionales que dificultaba notoriamente la intervención, oponiendo a unos contra otros y devaluando sistemáticamente la ayuda ofrecida, siendo éste un patrón que ya había sido notado por equipos anteriores.

\section{Preparación e Indicación}

El proceso de reunificación familiar plantea una serie de etapas que dieron forma al caso antes de indicarse el uso de IG: (1) la primera etapa, implicó un acercamiento paulatino entre la madre y el niño aún institucionalizado, ampliándose cada vez más el tiempo de visitas del niño a su hogar; (2) la segunda etapa, correspondió al egreso de Felipe de la Residencia y su retorno definitivo a su familia, siendo apoyados profesionalmente, mediante intervención familiar psicosocial, en las primeras semanas de reunificación; (3) la tercera etapa, correspondió al ingreso de la familia al programa de Familias de Acogida, donde el equipo de psicólogos y asistentes sociales evaluaron el caso. En esta tercera etapa, la desvinculación entre la madre y sus hijos, sobre todo de Felipe, generada por los años de institucionalización, aunado a sus carencias en el plano material y sus dificultades para cuidarlos, evidenció la necesidad de coordinarse con otros profesionales en la búsqueda de las múltiples soluciones que esta familia requería.

Así, (4) la cuarta etapa correspondió a una serie de gestiones sociales realizadas desde el programa, en dos líneas centrales: (a) con la madre se definió la entrega de un apoyo material y económico mensual, visitas domiciliarias de apoyo a sus competencias parentales y su integración a un grupo de madres del programa como red social de apoyo; y (b) con Felipe, se realizó un trabajo coordinado con la profesora del colegio, quien ya había realizado interconsulta para que Felipe fuera evaluado por un Neurólogo, concretándose esta derivación durante el proceso de IG. Todo esto ilustra que la IG se indicó sobre la base de una plataforma sólida de otras intervenciones psicosociales, que permitieron a los terapeutas concentrarse en el foco definido y planificar acciones clínicas acotadas a dicho foco.

Finalmente, (5) la quinta etapa corresponde a la intervención clínica propiamente tal, siendo ésta una necesidad que surge de la información aportada por las etapas previas. Es así como la intervención psicosocial realizada, permitió detectar en conjunto con otros profesionales la presencia de un Trastorno por Déficit de Atención con Hiperactividad en Felipe (DSM-IV-TR, 2002), ligado a conductas disruptivas, agresivas e impulsivas, que se manifestaban en la casa y el Colegio. A su vez, Sara presentaba un Trastorno Narcisista de Personalidad (DSM-IV-TR, 2002), lo cual complejizó aún más la intervención. A nivel familiar, se observó conflictos paterno-filiales y fraternales.

Tomando en cuenta estos antecedentes, el equipo de Familias de Acogida decidió indicar el uso de Interacción Guiada a Sara y sus hijos, por cuanto se valora como una terapia que permite trabajar en un área específica, en este caso: el vínculo de la madre con sus hijos en un proceso de reunificación familiar. Respecto al trastorno presentado por la madre, se evaluó que el deterioro de la empatía no era lo suficientemente severo como para impedir un trabajo de vinculación familiar.

\section{Primera Sesión de la $I G$}

Participan la madre, sus dos hijos y los dos terapeutas a cargo de la intervención. Se disponía de una sala amplia, con una alfombra, una mesa y sillas cómodas, una caja con una variedad de juguetes acordes a la edad de los niños, además de una cámara de videograbación fija.

Luego de la presentación inicial, se indaga sobre la incorporación reciente de Felipe al grupo familiar. Tanto Sara como Alicia casi al unísono responden: "lo queremos y punto... y punto". Alicia agrega que "Felipe es mиy gritón y desobediente, hay que estar siempre llamándole la atención".

Se inicia la primera entrevista con la familia, en que se busca aclarar el encuadre de esta nueva intervención y definir los roles y expectativas respecto a la terapia. Sara se refiere en forma crítica y negativa hacia la intervención anterior, planteando que se aburrían y que esta intervención "no sirvió de nada". Esto es comprendido desde las características de personalidad de la madre, manifestadas en un patrón histórico de sistemática devaluación de la ayuda profesional recibida.

Se le explica claramente a la familia las características específicas de esta modalidad de intervención, destacando su rol como protagonista del proceso y enfatizando que su participación es muy importante para el logro de resultados exitosos. Una vez que la familia se muestra dispuesta a "probar" esta nueva metodología, se firma el consentimiento informado, y se procede a señalar la siguiente 
consigna adaptada de Robert-Tissot y Cramer (1998; en Forcada-Guex, 2007):

"Vamos a proponerle que juegue libremente con sus hijos durante 15 minutos. Nosotros sólo observaremos sin participar. Grabaremos el juego en video, para ver con usted algunos momentos en la próxima sesión. Esto podría ayudarle a entender y mirar de forma diferente los comportamientos de sus hijos y la manera con la cual usted responde a ellos. Trataremos entonces de hacer un vínculo con las dificultades que usted tiene ahora con sus hijos".

Luego, se realiza la filmación de 15 minutos de juego. En síntesis, se pueden diferenciar seis etapas en el desarrollo del juego familiar: (1) Fase de exploración de los juguetes; (2) Construcción de una casa, juegan Sara y Alicia principalmente, Felipe se mantiene excluido; (3) Los tres logran generar un juego coordinado en la escenificación de la "caperucita roja" con títeres (Felipe es el "lobo", Sara es el "cazador" y la "abuelita" y Alicia es la "caperucita roja"); (4) Juegos paralelos de cada miembro de la familia, sin mayor interacción entre ellos; (5) Sara y Alicia juegan juntas a armar una casa, Alicia mueve la mesa ("Terremoto") y la madre le llama la atención. Felipe lo hace a su vez, y Sara y Alicia lo descalifican irónicamente y (6) Término del juego, ordenan los juguetes.

Posteriormente, se le plantean a la Familia las preguntas preestablecidas por Forcada-Guex (2007):

¿Ha sido representativo este juego de lo que pasa en casa?

¿Algo le ha sorprendido?

¿Qué es lo que más le ha gustado? ¿Qué es lo que menos le ha gustado?

¿Piensa usted que su hijo(a) lo ha disfrutado? ¿Y usted?

Lo más significativo de los comentarios de la familia durante esta primera sesión de juego, es que la madre se cuestiona a partir de las preguntas realizadas: "nosotros nunca jugamos en la casa... ¿está mal que no juguemos?, ¿deberíamos hacerlo?".

Estos cuestionamientos generan una apertura en la madre a la posibilidad de desarrollar una relación más lúdica con sus hijos y a sentirse aliada a los terapeutas en relación a este objetivo particular.

Preparación de la siguiente sesión y selección de secuencias en relación al foco.

A partir de la primera entrevista, junto con el análisis de la filmación de juego libre, los terapeutas se plantean algunas hipótesis relacionales que permiten ir construyendo el foco a intervenir:

La madre y Alicia, al vivir más tiempo juntas, podrían estar aliadas contra Felipe ya que viene a alterar su dinámica relacional habitual (coalición como defensa mantenedora de la homeostasis familiar).
Alicia teme perder la relación que ha desarrollado con su madre (Felipe como amenaza a su relación de apego; fantasma de la internación ligada al temor de ser el tercero excluido).

Alicia probablemente ejerce roles parentales que la madre permite, como el tratar de controlar la conducta de Felipe (parentalización disfuncional).

Felipe al sentirse excluido del grupo familiar, podría actuar con rabia y manifestarlo sintomáticamente en conductas disruptivas (paciente índice, chivo emisario).

En base de estos antecedentes, se decide focalizar el tratamiento en fomentar una mayor sensibilidad materna hacia el proceso de reunificación, para lograr una inclusión adecuada de Felipe a la dinámica familiar.

Tomando en cuenta que existía un Trastorno Narcisista de Personalidad en Sara (DSM-IV-TR, 2002), se acuerda seleccionar tanto secuencias positivas como negativas (y no sólo positivas, lo que resulta adecuado por ejemplo en el caso de una madre depresiva) a mostrarse en la siguiente sesión. Esto se fundamentó en una tendencia observada en Sara a idealizarse como madre y en su dificultad para visualizar aspectos a mejorar en la relación con sus hijos, por lo que se acuerda mostrarle secuencias en que la madre aparece en primer lugar incluyendo a Felipe en el juego, para luego confrontarla con imágenes en las cuales lo excluye. En la Tabla 2 se presentan las etapas del juego y las secuencias positivas y negativas seleccionadas de acuerdo al foco por los terapeutas.

Se consideró que si bien era importante empoderarla y reforzarla como madre, también era necesario que ella visualizara y verbalizara aspectos de la relación que podían ser vistos como problemáticos, para que éstos pudieran ser captados por ella. La siguiente sesión, entonces, se planificó como un espacio para abordar reflexivamente la tensión entre ambos aspectos.

\section{Segunda Sesión de la IG}

Participan la madre y los dos terapeutas. Se vuelve a abordar el tema de la frustración y sensación de inutilidad respecto de la intervención anterior y se le señalan las expectativas que se tienen de ella en relación a su participación activa para lograr avances en la psicoterapia.

Se comienza con la revisión de las secuencias positivas en las que la madre incluye a Felipe en la dinámica de juego (ver Tabla 2, Secuencias Positivas I, IV, V, VI y VII) o responde sensiblemente a las necesidades de ambos (ver Tabla 2, Secuencias Positivas II, III).

Una vez que se observa cada secuencia, se comienza con la fase de preguntas en relación a dicha secuencia. Se le solicita en primer lugar que describa lo que observa, luego se la lleva a ponerse en el lugar de Felipe en la situación lúdica y a empatizar con sus sentimientos. Posteriormente, 
Tabla 2: Etapas del Juego, Secuencias Positivas y Secuencias Negativas Seleccionadas

\begin{tabular}{|c|c|c|}
\hline Etapa del Juego & Secuencia $(+)$ & Secuencia (-) \\
\hline \multicolumn{3}{|l|}{ (1) Exploración } \\
\hline \multirow{2}{*}{$\begin{array}{l}\text { (2) Construcción de una } \\
\text { casa }\end{array}$} & Secuencia-I: 1’00” & Secuencia-I: 1’10” \\
\hline & Madre incorpora a Felipe en el juego, ambos ríen. & $\begin{array}{l}\text { Felipe, luego de un rato de sentirse ignorado por Sara, } \\
\text { se queja y hace una pataleta de breve duración. } \\
\text { S II: } 2 \text { ' } 25 \text { " } \\
\text { Felipe habla usando el juguete del dinosaurio y no es } \\
\text { tomado en cuenta; Mamá ofrece algo a Alicia; Felipe se } \\
\text { frustra y se lo quita con violencia. }\end{array}$ \\
\hline \multirow[t]{7}{*}{ (3) Caperucita Roja } & S II: 4’50” & S III: 3’54" \\
\hline & $\begin{array}{l}\text { Finalmente madre considera la propuesta de Alicia. } \\
\text { S III: 5'34" }\end{array}$ & $\begin{array}{l}\text { Sara rechaza una propuesta de Alicia. Alicia se siente } \\
\text { ignorada y su rostro muestra incomodidad. }\end{array}$ \\
\hline & Madre acepta cambio de juego de Alicia. Felipe & $S I V: 4^{\prime} 22^{\prime \prime}$ \\
\hline & $\begin{array}{l}\text { se adapta bien y juegan ambos hermanos, madre } \\
\text { acompaña y ayuda en forma no intrusiva, dejando la } \\
\text { iniciativa a los niños. } \\
S \text { IV: } 6^{\prime} 08^{\prime \prime}\end{array}$ & $\begin{array}{l}\text { Alicia propone jugar, madre no contesta y no sigue el } \\
\text { juego. Alicia debe completar sola el juego y reclama. }\end{array}$ \\
\hline & $\begin{array}{l}\text { Felipe se adelanta y se come a la abuela. Madre lo } \\
\text { acepta bien y sigue el juego. } \\
S V: 6^{\prime} 34^{\prime \prime}\end{array}$ & \\
\hline & Sara apoya a Felipe y logra fomentar el juego entre & \\
\hline & los tres. & \\
\hline \multirow[t]{2}{*}{ (4) Juegos Paralelos } & $S$ VI: $8^{\prime} 45^{\prime \prime}$ & $S V: 10^{\prime} 23 "$ \\
\hline & $\begin{array}{l}\text { Auto de bomberos, ella le ofrece jugar a Felipe, quien } \\
\text { se muestra feliz. }\end{array}$ & $\begin{array}{l}\text { Sara no le responde a Felipe en cuatro oportunidades. } \\
\text { Felipe se aleja. }\end{array}$ \\
\hline \multirow[t]{2}{*}{ (5) Terremoto } & & $S$ VI: $12 ׳ 30 ”$ \\
\hline & & $\begin{array}{l}\text { Sara nuevamente ignora a Felipe, quien juega solo. Felipe } \\
\text { descubre que moviendo la mesa y botando los juguetes } \\
\text { puede llamar la atención de la madre. Se genera una } \\
\text { alianza de Sara y Alicia, quienes se burlan de Felipe. }\end{array}$ \\
\hline \multirow[t]{2}{*}{ (6) Término de los juegos } & $S$ VII: $13^{\prime} 20^{\prime \prime}$ & \\
\hline & $\begin{array}{l}\text { Madre incorpora amistosamente a Felipe en la } \\
\text { tarea. }\end{array}$ & \\
\hline
\end{tabular}

se indaga en qué podría estar sintiendo ella misma en ese momento del juego.

La observación de las primeras secuencias seleccionadas produjo expresiones de gratificación en la madre (por ejemplo, ver el juego de la Caperucita Roja, secuencia positiva III y en los Juegos Paralelos, secuencia positiva VI) y permitió a los terapeutas trabajar sobre aspectos conductuales concretos de la interacción, usando como guía los criterios propuestos por Crittenden (1981, en Forcada-Guex, 2007): (1) Expresiones faciales; (2) Expresiones vocales; (3) Posición y el contacto corporal; (4) Expresiones de afecto; (5) Ritmo de la toma de los roles; (6) Control; (7) Tipo de actividad.

Posteriormente, se le muestran algunas de las secuencias negativas en relación al foco (ver Tabla 2, secuencias negativas), trabajándolas del mismo modo que las interacciones positivas, de manera que la madre pudiese percibir la diferencia y discutir sobre cómo ella podía generar una interacción más positiva con sus hijos.
Dicha observación permitió a la madre expresar las proyecciones negativas hacia Felipe (ver Tabla 2, secuencia negativa I) que subyacían a la interacción: "Felipe es muy parecido a Osvaldo, su papá, son iguales físicamente, cada vez que Felipe se pone agresivo es como ver a su papá”.

\section{Síntesis del proceso terapéutico (sesiones 3 a 9)}

En las siguientes sesiones se continuó trabajando de la misma forma en que las dos primeras, intercalando sesiones de juego familiar con sesiones de retroalimentación "videada" con la madre, tal como se ilustró más arriba. Esta modalidad de trabajo permitió que Sara pudiera, a partir de las sesiones de observación de video, darse cuenta de los modos de interacción con sus hijos y así hacer más esfuerzos por incluir a Felipe al juego realizado en las sesiones. Esta dinámica de mayor inclusión se fue trasladando paulatinamente a las situaciones de la vida cotidiana en el hogar.

Asimismo, la revisión de los videos permitió expresar a Sara la dificultad para manifestar cariño físico a Alicia, y 
los sentimientos ambivalentes asociados: “...es como era yo con mi madre, no me gusta que sea tan arrastrada, que me ande como mendigando cariño". Estas declaraciones de la madre reflejaban un vínculo con su propia historia, en tanto la relación con su hija le recordaba la historia vincular con su propia madre, en que Sara se sentía fuertemente rechazada por ella. Tanto este tema, como las proyecciones negativas hacia Felipe, se abordaron conectando elementos relevantes de su historia con los patrones interaccionales actuales de Sara con sus hijos.

Estos patrones eran observables en los fragmentos de video que se le mostraban, y las intervenciones clínicas se dirigían a ayudarla a diferenciar ambos planos, destacando sus momentos de logro y competencia (p.ej., “ ¿ve que se puede hacer de otra forma? Usted tomó en cuenta el juego de Felipe sin dejar de lado a Alicia... eso es un avance notable") y permitiendo "limpiar" de esta forma su relación actual de los "fantasmas" del pasado.

Cabe enfatizar que esto se logró mediante la técnica de "iluminar" ciertos aspectos de la interacción en el juego durante todo el proceso (p.ej., pausando el video, retrocediendo y repitiendo la secuencia dos o tres veces), y usando preguntas abiertas que pacientemente acompañaban la elaboración que la madre hacía de los temas propuestos (p.ej., “'Eso fue un momento difícil del juego!... ¿Qué cree usted que estaría pasando con Felipe en ese momento?... ¿y con Alicia?”).

Un elemento relevante de este proceso fue que dicha conversación se usó para mejorar la sensibilidad de Sara a las señales comunicativas de sus hijos, trabajando en primer lugar la detección de éstas y luego sobre su interpretación de dichas señales.
Como consecuencia de lo anterior, la madre empezó a desarrollar una relación más lúdica con sus hijos, realizando más actividades juntos y disfrutando más la relación con ellos.

\section{Cierre de la intervención.}

Al finalizar la terapia, los miembros de la familia mostraron mejorías en su interacción, visualizadas tanto en el análisis de los videos como señaladas por la madre. Respecto al análisis de las sesiones de video se pudo observar de manera cualitativa los indicadores señalados en la tabla 3 .

La madre valoró el hecho de que la técnica empleara el uso de videos para poder verse a sí misma interactuando con sus hijos, como una ayuda importante para mejorar su modo de relacionarse con ellos y el poder sentirse parte y comprender el sentido de la psicoterapia.

Se decidió finalizar el proceso en conjunto con la madre, debido a que las principales dificultades relacionales con sus hijos se habían resuelto, habiendo mejorado su sensibilidad materna y la interacción con ambos.

Felipe mostró mejorías importantes en sus conductas disruptivas en el colegio, obteniendo avances en su adaptación escolar reconocidas por sus profesores. Estos resultados, si bien se obtuvieron durante el proceso de IG, también se deben a la oportuna derivación a neurólogo y el apoyo farmacológico de Metilfenidato durante el horario escolar, además del apoyo mostrado por la profesora jefe de Felipe. Cabe destacar que Felipe mantuvo sus cambios conductuales en el hogar aun sin el efecto del medicamento, visualizándose la interacción fármaco-IG como coadyuvantes en el proceso desarrollado.

Tabla 3: Resultados Obtenidos en la Intervención

Resultados de la IG obtenidos en la psicoterapia

(a través del análisis cualitativo de los videos de las sesiones de juego)

En el cuidador/a
En los niños

En el sistema familiar

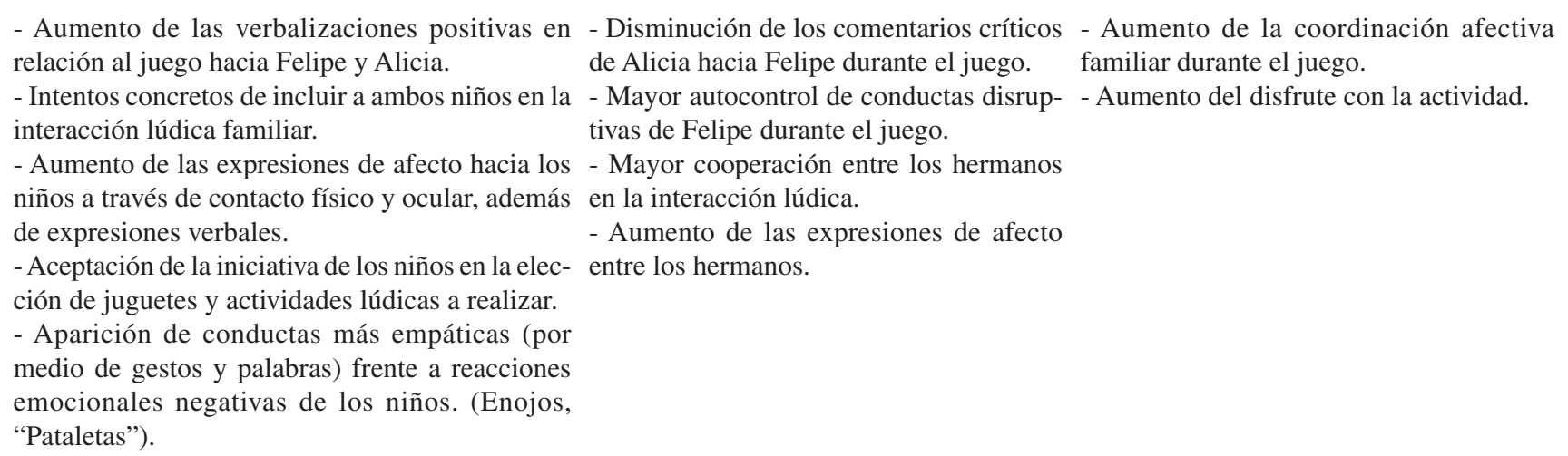


Alicia ya no ejercía de manera directa un rol parental con Felipe y Sara estaba más posicionada como madre de ambos niños y disfrutaba mucho más de su relación con ellos. Finalmente, se acordó realizar un seguimiento mensual para reforzar logros del proceso de psicoterapia.

\section{Discusión}

Se han expuesto los fundamentos y principios en que se sustenta la Terapia de Interacción Guiada, detallando sus objetivos, características, metodología, indicaciones y resultados demostrados, ilustrando estos elementos mediante la exposición de un caso clínico exitoso desarrollado en un programa social de la Sociedad Protectora de la Infancia.

El modelo de IG implementado en la institución se construyó a partir de los postulados de McDonough (2004), Rusconi-Serpa (1992) y Robert-Tissot, et al. (1996). Esta integración se realizó con el objetivo de recoger los aspectos de cada uno de estos enfoques que más se adapten al contexto chileno de intervención en programas sociales.

Respecto a las ventajas de esta modalidad de intervención, uno de los beneficios observados es que permite al terapeuta preparar la sesión de retroalimentación videada en forma previa, a partir del trabajo de selección de secuencias que faciliten los cambios conductuales buscados en el padre o la madre, de acuerdo al foco establecido para el tratamiento. Así, es posible facilitar tanto la gestión del terapeuta como la supervisión del caso y ensayar previamente el modo específico de intervención.

Además, la opción de citar solamente a los adultos significativos participantes del proceso a las sesiones de revisión de secuencias, permite abordar temáticas que podrían resultar más difíciles de tratar en presencia de los niños, sobre todo al considerar que se trata de familias multiproblemáticas, en las cuales emergen temáticas de alto contenido traumático. Por ejemplo, hemos visto que la IG permite profundizar en elementos de la historia de la madre y relacionarlos con las dificultades de la interacción actuales (ver caso clínico). El disponer de un espacio a solas con el o los adultos involucrados ha facilitado vincular ambos aspectos en la discusión de las secuencias, generando una mayor consciencia de las distorsiones de la interacción y comunicación con sus hijos.

Otra de las ventajas que presenta la IG, es que en los casos de padres o cuidadores que se han sentido excluidos en la toma de decisiones, por parte de los programas sociales sobre las intervenciones de las cuales son foco ellos y sus hijos, la IG les permite sentirse protagonistas y agentes del cambio debido al gran énfasis que pone en los aspectos positivos de la interacción y al ir involucrando progresivamente a los padres en el trabajo respecto al foco y la planificación de las sesiones de juego. Como consecuencia de esto, los padres tienden a visualizar al terapeuta como un aliado que los empodera para ayudar de mejor manera a sus hijos(as). El hecho de involucrar al padre o madre en la construcción de los aspectos conductuales del foco terapéutico, puede contribuir a un cambio de paradigma tanto en los usuarios como en los profesionales y pasar de un enfoque centrado en el problema a un enfoque centrado en la solución (Sousa, Ribeiro \& Rodrigues, 2006), considerando al usuario como un agente activo y no como un receptor pasivo de ayuda social. Es decir, la IG genera una motivación en los usuarios, produciendo "enganches" en algunas personas que se muestran resentidas por malas experiencias y por lo tanto reticentes a las intervenciones psicológicas y faltos de motivación (McDonough, 1993; 2004). En esto, la modalidad de juego es esencial al introducir un elemento novedoso para los padres y niños/as, generando un espacio donde el aspecto lúdico de la relación es lo central. Para muchas familias, esto significa construir una nueva forma de compartir e interactuar con sus hijos/as. La situación de juego en familia, según lo que señalan los usuarios de la Sociedad Protectora de la Infancia, les resulta muy gratificante y entrega una experiencia de relación nueva y diferente. Como se ha señalado anteriormente, las familias usuarias son en general multiproblemáticas, en las cuales es frecuente observar una carencia de dinámicas de juego familiar. Los padres, entonces, se sorprenden de que este pequeño espacio de interacción lúdica les permita, con la ayuda del terapeuta, descubrir y comprender aspectos no visualizados de la interacción con sus hijos.

A partir de lo anteriormente expuesto, es posible sostener que la IG constituye una herramienta de intervención de gran utilidad tanto en los programas sociales como en los servicios públicos de salud mental (cuyos usuarios corresponden en un alto porcentaje a familias multiproblemáticas), al ser una intervención breve, focalizada y que puede ser implementada en paralelo y de manera complementaria a otras intervenciones tanto familiares como de los miembros de la familia de forma individual. Así, casos en que la madre está siendo intervenida individualmente, por ejemplo por depresión con fármacos y psicoterapia, puede iniciar un proceso de IG para mejorar los aspectos de la interacción con sus hijos. En este sentido, el potencial de la IG para trabajar en casos que muestren dificultades en los vínculos de apego, sea como foco principal de abordaje o como una temática relacionada significativa para el avance del proceso, se muestra como una aplicación prometedora para los programas sociales.

Se destaca que los beneficios de una aplicación rigurosa, sobre el trasfondo de una sólida alianza terapéutica y una plataforma de intervenciones complementarias, han mostrado un impacto relevante sobre los usuarios atendidos con IG en Familias de Acogida, generando en los cuidadores: (a) un aprendizaje enriquecido sobre la interacción con sus hijos y el despliegue de sus habilidades parentales, y (b) una 
transferencia conductual de estos aprendizajes a las sesiones de juego familiar y, posteriormente, a la vida cotidiana.

Por último, cabe mencionar que para trabajar la interacción guiada es necesario que los terapeutas cuenten con un entrenamiento clínico previo, de manera que pueda ser indicada y aplicada con criterio de especialista en psicoterapia, ya que esta modalidad técnica es un medio para trabajar una mejor vinculación entre los padres y sus hijos, no un fin en sí misma. La IG, aplicada como se ha mostrado en este artículo, permite la apertura de aspectos delicados del pasado del cuidador, por lo que se requiere de habilidades clínicas para el manejo adecuado de estos temas. Es por esto que en el contexto chileno resulta un desafío relevante la inversión en capacitación y especialización de los profesionales de los programas sociales, para así responder a la compleja demanda de las familias multiproblemáticas y en riesgo social de nuestro país.

\section{Referencias}

American Psychiatric Association (2002) DSM-IV-TR. Manual diagnóstico y estadístico de los trastornos mentales. Texto revisado. Barcelona: Editorial Masson.

Bakermans-Kranenburg, M, van IJzendoorn, M. \& Juffer, F. (2005). Disorganized Infant Attachment and Preventive Interventions: a Review and Meta-Analysis. Infant Mental Health Journal, 26(3), 191-216.

Bakermans-Kranenburg, M., Juffer, F. \& van IJzendoorn, M. (1998). Interventions with Video Feedback and Attachment Discussions: Does Type of Maternal Insecurity Make a Difference? Infant Mental Health Journal, 19, 202-219.

Beebe, B. (2003). Brief mother-infant treatment: psychoanalytically informed video feedback. Infant Mental Health Journal, 24(1), p. 24-52

Benoit, D., Madigan, S., Lecce, S., Shea, B., \& Goldberg, S. (2001). Atypical maternal behaviour toward feeding-disordered infants before and after intervention. En Infant Mental Health Journal, 22(6), 611-626.

Bromwich, R. (1990). The Interaction Approach to Early Intervention. En Infant Mental Health Journal, 11, 66-79.

Cunningham, P. \& Henggeler, S. (1999). Engaging Multiproblem Families in Treatment: Lessons Learned Troughout the Development of Multisystemic Therapy. Family Process, 38, 265-281.

Fonagy, P., Steele, M., Steele, H., Leigh, T., Kennedy, R., Mattoon, G. \& Target, M. (1995a). Attachment, the reflective self and borderline states: The predictive specificity of the Adult Attachment Interview and pathological emotional development. En: S. Goldberg, R. Muir \& J. Kerr (eds). Attachment Theory: Social, Developmental and Clinical Perspectives, p. 233-278. Hillsdale, NJ: The Analytic Press.

Forcada-Guex, M. (2007, junio) Interacciones Guiadas: Aplicaciones Preventivas y Clínicas. Seminario-Taller organizado por el Diplomado Promoción de Apego Seguro: Enfoque interdisciplinario en la construcción del primer vínculo, Pontificia Universidad Católica, Santiago, Chile.

Forsyth, J., Kennedy, H. \& Simpson, R. (1996). Video interaction guidance in schools - we've looked at life from both sides now. Dundee Educational Psychology Service.

Forsyth, P. \& Simpson, R. (2002) If you've got it do you flaunt it?: the use of the Video remote control in Video Interaction Guidance Feedback. Dundee Educational Psychology Service.

Gómez, E., Muñoz, M. \& Haz, A.M. (2007). Familias Multiproblemáticas y en Riesgo Social: Características e Intervención. Psykhe, 16, 43-54

Juffer, F., Bakermans-Kranenburg, M. \& van IJzendoorn, M. (2005). Enchancing Children's Socio-Emotional Development: A Review of
Intervention Studies. En: Teti, D.M. (Ed) (2005). Handbook of Research Methods in Developmental Science, p. 213-232. Oxford: Blacwell.

Karlsson, K. \& Skagerberg, A. (1999) Experience from the floor of attachment and relationship building. The Signal, 7, 1-7

Kivijärvi, M., Räihä, H., Virtanen, S., Lertola, K. \& Piha, J. (2004). Maternal sensitivity behavior and infant crying, fussing and contented behavior: The effects of mother's experienced social support. Scandinavian Journal of Psychology, 45, 239-246.

Larrieu, J. \& Zeanah, C. (2004). Treating Parent-Infant Relationships in the Context of Maltreatment: An Integrated Systems Approach. pp. 243-264. En: Sameroff, A. J., McDonough, S. C. \& Rosenblum, K. L. (Eds) (2004). Treating Parent-Infant Relationship Problems: Strategies for Intervention. New York: The Guilford Press.

Lieberman, A. (2004). Child-Parent Psychotherapy: A Relationship-Based approach to the Treatment of Mental Health Disorders in Infancy and Early Childhood. pp. 97-122. En: Sameroff, A. J., McDonough, S. C. \& Rosenblum, K. L. (Eds) (2004). Treating Parent-Infant Relationship Problems: Strategies for Intervention. New York: The Guilford Press.

McDonough, S. (1993). Interaction guidance: Understanding and treating early infant-caregiver relationship disturbances. pp 414-426. En: Zeanah, C. H. (Ed) (1993). Handbook of Infant Mental Health. New York: The Guilford Press.

McDonough, S. (1995). Promoting positive early parent-infant relationships trough interaction guidance. Child and Adolescent Psychiatric Clinics of North America, 4, 661-672.

McDonough, S. (2004). Interaction guidance: promoting and nurturing the caregiving relationship. pp. 79-96. En: Sameroff, A. J., McDonough, S. C. \& Rosenblum, K. L. (Eds) (2004). Treating Parent-Infant Relationship Problems: Strategies for Intervention. New York: The Guilford Press.

McNeil, C. \& Herschell, A. (1998). An essay for practitioners. Treating Multi-Problem, High Stress Families: Suggested Strategies for Practitioners. Family Relations, 47, 259-262.

Oppenheim, D. Goldsmith, D. \& Koren-Karie, N. (2004). Maternal insightfulness and preschoolers' emotion and behaviour problems: Reciprocal influences in a therapeutic preschool program. Infant Mental Health Journal, 25, 352-367.

Protectora de la Infancia (2007). Memoria Anual 2006. Santiago de Chile: Sociedad de Asistencia y Capacitación.

Robert-Tissot, C., Cramer, B., Stern, D. S., Serpa, S. R., Bachmann, J. P., Palacio-Espasa, F., Knauer, D., De Muralt, M., Bernay, C. \& Mendiguren, G. (1996). Outcome evaluation in brief mother-infant psychotherapies: Report on 75 cases. Infant Mental Health Journal, 17, 97-114.

Rusconi-Serpa, S. (1992). La guidance interactive : Les points essentiels du traitement. Psychoscope, 10, 7-10.

Schechter, D. S. (2004) How post-traumatic stress affects mothers' perceptions of their babies: A brief video feedback intervention makes a difference. Zero to Three, 24(3), 43-49.

SENAME (2007). Catastro de la Oferta Programática de la Red SENA$M E$. Santiago: Departamento de Planificación y Control de Gestión del Servicio Nacional de Menores.

Sharlin, S. \& Shamai, M. (1995). Intervention with Families in Extreme Distress (FED). Marriage \& Family Review, 21, 91-122.

Simpson, R. (2001). Video Interaction Guidance in Parenting and Family Focused Work. Association of Child Psychology and Psychiatry. Occasional Papers No.18.

Sousa, L., Ribeiro, C. \& Rodrigues, S. (2006). Intervention With Multiproblem Poor Clients: Towards a Strengths-Focused Perspective. Journal of Social Work Practice, 20, 189-204.

Svanberg, P. O. G. (2005) Promoting attachment security in primary prevention using video-feedback: The Sunderland Infant Programme. Infant Mental Health Journal. En prensa.

Vereijken, C., Riksen-Walraven, M. \& Kondo-Ikemura, K. (1997). Maternal Sensitivity and Infant Attachment Security in Japan: A Longitudinal Study. International Journal of Behavioral Development, 21, 35-49. 
